\title{
Can the Dustiest Main Sequence Stars Tell Us About the Rocky Planet Formation Process?
}

\author{
Carl Melis \\ Center for Astrophysics and Space Sciences, University of California, San Diego, California \\ 92093-0424, USA \\ email: cmelis@ucsd.edu
}

\begin{abstract}
Main sequence stars hosting extreme quantities of inner planetary system debris are likely experiencing transient dust production events. The nature of these events, if they can be unambiguously attributed to a single process, can potentially inform us on the formation and/or early evolution of rocky Earth-like planets. In this contribution I examine some of the dustiest main sequence stars known and three processes that may be capable of reproducing their observed properties. Through this activity I also make an estimate for the likelihood of an A-type star to have an asteroid belt-like planetesimal population.
\end{abstract}

Keywords. planets and satellites: formation, circumstellar matter, planetary systems

\section{Introduction}

Understanding how rocky terrestrial planets form and evolve is paramount to the question of how life forms on planets and whether or not other sentient beings can exist in the universe. Currently the best means of identifying stars undergoing terrestrial planet formation or collisional evolution events is discovery and characterization of dusty debris disks that orbit in the inner planetary system of their host star (e.g., Kenyon \& Bromley 2006; Melis et al. 2010; Jackson \& Wyatt 2012; Meng et al. 2014, 2015; Genda et al. 2015). Dust at a distance of $\sim 1$ AU from a Sun-like star will be heated to Earth-like temperatures, $\sim 300 \mathrm{~K}$, and will emit in the mid-infrared, thus most surveys for inner planetary system dust look for excess emission at wavelengths of $10-20 \mu \mathrm{m}$.

Investigations into how mid-infrared excess stars provide insight into the rocky planet formation process are being actively pursued by several groups. Statistical works conducted to date typically do not discriminate on how dusty a given debris disk system is when including it in their analysis (e.g., Meyer et al. 2008; Jackson \& Wyatt 2012; Genda et al. 2015). It is worth noting that it need not be the case that all mid-infrared excesses are linked to rocky planet formation events. Indeed, another hypothesis is that weaker excesses can be attributed to dust produced through collisional evolution of asteroid belt-analogs. Such a suggestion seems quite appropriate for the Meyer et al. (2008) sample which covers an age range of 0.3-3.0 Gyr where one would not expect rocky planet formation collisional events to be occurring. As such, one might interpret the Meyer et al. (2008) statistics as telling us that $19-32 \%$ or up to $62 \%$ of Sun-like stars host asteroid belt analogs. Under this banner, I estimate the occurrence rate for asteroid belts around A-type stars.

Examination of the incidence rate of such systems at young ages (10-30 Myr old), when collisional cascade dust production is at its height (e.g., Wyatt 2008), should enable a reasonable estimate of the fraction of stars that host asteroid-belt analogs. To ensure a robust estimate, it is important to use a statistical sample of stars observed in a 
blind survey where it is clear that the mid-infrared excess emission originates from inner planetary system material. Morales et al. (2009) provide just such a data set by combining Spitzer MIPS and IRS measurements for relatively luminous stars. Their data set, which probes stars of spectral type A and late-B that were previously determined to have excess emission at $24 \mu \mathrm{m}$, shows that 10 out of 14 (or $71 \%$ ) of 24 and $70 \mu \mathrm{m}$ detected stars have blackbody-fit dust temperatures $\gtrsim 200 \mathrm{~K}$ (only those stars with single-temperature blackbody fits that are detected at 24 and $70 \mu \mathrm{m}$ are selected to ensure that the dust temperature - and hence physical location - is well constrained). All of these 10 sources have a fractional infrared luminosity that is $<2 \times 10^{-4}$ and thus can be interpreted as hosting active asteroid belt analogs (similar to the conclusion reached by Morales et al. 2009). Rieke et al. (2005) and Su et al. (2006) present unbiased $24 \mu \mathrm{m}$ excess statistics, and each find that roughly $40-50 \%$ of A-type stars with ages between 10-30 Myr host $24 \mu \mathrm{m}$ excess emission. When combined with the finding from Morales et al. (2009) that $\approx 71 \%$ of A-type stars with $24 \mu \mathrm{m}$ excess emission have dust in their inner planetary system, it is found that at least $33 \%$ of A-type stars with age in the range of 10-30 Myr should host an active planetesimal belt in their inner planetary system. This occurrence rate is in good agreement with the statistics of polluted white dwarf stars discussed by Zuckerman et al. (2003) and Zuckerman et al. (2010), indicating that such asteroid belt-analogs go on to eventually pollute their dead stars' atmospheres.

Rather than working with all mid-infrared excess main sequence stars, one might instead opt to examine only those hosting extreme quantities of inner planetary system material as they are likely experiencing transient dust production events. To date, only a handful of main sequence stars are known to have sufficiently high levels of mid-infrared excess emission (and hence inner planetary system dust) that points to an unambiguous origin in transient processes that may be relevant to terrestrial planet formation or evolution (e.g., Song et al. 2005, Rhee et al. 2007, Rhee et al. 2008, Zuckerman et al. 2012, Melis et al. 2012, Olofsson et al. 2012, Schneider et al. 2013, Melis et al. 2013, Kennedy \& Wyatt 2014). Melis et al. (2014) describe how to sort inner planetary system debris disks into two general categories: those that result from collisional grinding down of a population of rocky planetesimals (an active asteroid belt-analog) and those that require a transient dust production event (stochastic or transient collisions involving planetary-scale objects). They begin with the assumption that all dust disks are the product of collisions of numerous small rocky bodies in an active planetesimal belt. It is then estimated what total mass of parent bodies $-M_{P B}-$ is necessary to reproduce the observed parameters for the small dust grains and compare $M_{P B}$ to the model simulations of Kenyon \& Bromley (2006) for the formation of terrestrial planets to see if this amount of mass should have coalesced to form planetary embryos or planets. Should the indicated mass of parent bodies be sufficient to expect the formation of planetary-scale bodies $\left(M_{P B} \gtrsim 1 \mathrm{M}_{E \text { arth }}\right)$, then it is concluded that the active planetesimal belt hypothesis is false (as any planetesimal population should have developed into planetary embryos or planets) and that the observed dust results from transient dust-production involving large rocky objects.

\section{Extreme debris disk systems}

All main sequence stars currently known and confirmed to be hosting substantial quantities of inner planetary system dust - and thus likely to be undergoing transient dust production events $\left(\mathrm{M}_{P B} \gtrsim 1 \mathrm{M}_{\text {Earth }}\right)$ - are presented in Figure 1. Examination of the properties of these systems reveals that those with early-type host stars are mostly observed when the star is $\sim 10$ Myr old. By contrast, this epoch extends later for Sun-like 

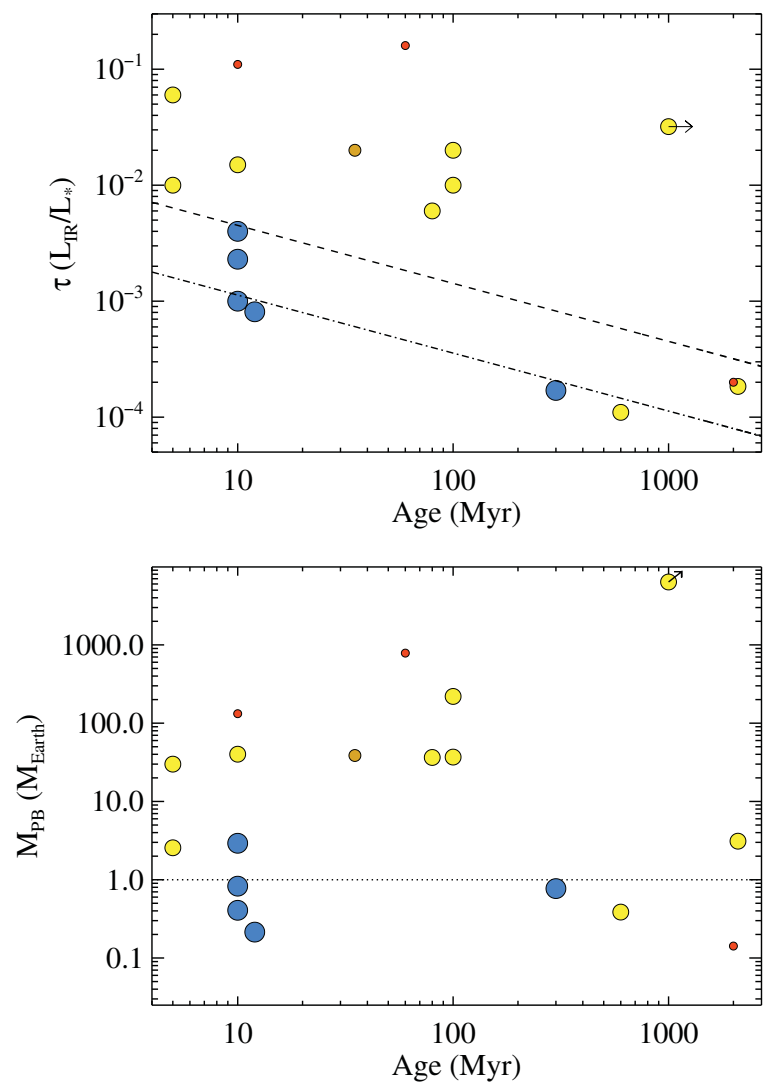

Figure 1. Both Panels: Stars hosting large quantities of inner planetary system material likely generated in transient dust production events $\left(\mathrm{M}_{P B} \gtrsim 1 \mathrm{M}_{E \text { arth }}\right.$; see Melis et al. 2014). Objects down to $0.1 \mathrm{M}_{\text {Earth }}$ are still considered as candidates but could be explained by collisional grinding down of an active planetesimal belt; some representative systems are shown. Sources are drawn from Melis et al. (2010), Melis et al. (2013), and references therein. Small, red circles are K-type stars. The medium-small, gold circle is a G-type star. Medium-large, yellow circles are F-type stars. Large, blue circles are A-type stars. The object with a limit arrow and age of $1000 \mathrm{Myr}$ is BD+20 307 as its age is a lower limit. Upper Panel: The dashed line represents $\tau$ necessary to realize a parent body mass of $1 \mathrm{M}_{\text {Earth }}$ for a star of solar mass, radius, and temperature that is orbited by $300 \mathrm{~K}$ dust. The dash-dotted line represents the $\tau$ necessary to realize a parent body mass of $1 \mathrm{M}_{E \text { arth }}$ for a star of $1.7 \mathrm{R}_{\odot}, 2.0 \mathrm{M}_{\odot}$, and $8180 \mathrm{~K}$ effective temperature that is orbited by $300 \mathrm{~K}$ dust. Lower Panel: The dotted line represents the cutoff above which the observed dust is expected to be the result of transient processes (see Melis et al. 2014).

stars with observed specimens typically having ages $\leqslant 100 \mathrm{Myr}$. The incidence rate of the dustiest Sun-like stars, determined by Melis et al. (2010) to be roughly 1 in 300 for the age range of 30 to $100 \mathrm{Myr}$, is comparable to that for early-type stars of approximately 1 in 200 for the age range of 10 to $20 \mathrm{Myr}$ (Melis et al. in preparation). If all stars undergo an exceptionally dusty phase of inner planetary system evolution, then the observable signature of it should persist for about $1.5 \times 10^{5}$ years for Sun-like stars (Melis et al. 2010) and $\sim 7 \times 10^{4}$ years for early-type stars (Melis et al. in preparation).

To be consistent with the data presented in Figure 1, any model should reproduce the high level of dust observed around each star, the dominant timescale in which this dust is observed, and the observed incidence rate. Three possibilities are presented below. 


\subsection{Giant Impacts}

Rhee et al. (2008) and Melis et al. (2010) interpret the incidence rate of the dustiest main sequence stars with the aid of colliding planetary embryo models developed in Agnor et al. (1999), Agnor \& Asphaug (2004), and Asphaug et al. (2006). In brief, following a giant impact of two rocky planetary embryos or planets, fragmented debris covering a range of sizes is released. The collision time for the smallest dust particles produced in the ensuing collisional cascade will be about 1 year divided by $80 \times \mathrm{L}_{\mathrm{IR}} / \mathrm{L}_{\mathrm{bol}}$ (Melis et al. 2014 and references therein). If the largest initial fragments ejected during a giant impact have radii $\sim 100 \mathrm{~m}$ (see work by Agnor, Asphaug, and colleagues - note that this is in contrast to the suggestion by Jackson \& Wyatt (2012) and Genda et al. (2015) who suggest ejecta up to sizes of $500 \mathrm{~km}$ in diameter), then their collisional lifetime is $\sim 5 \times 10^{4}$ years for Sun-like stars and $\sim 2 \times 10^{4}$ years for early-type stars (see Melis et al. 2010). However, the formation of rocky terrestrial planets involves more than a single giant impact event (e.g., Stewart \& Leinhardt 2012). Through the analysis of Stewart \& Leinhardt (2012) and the simulations referenced therein it is found that a typical terrestrial planet will experience $\sim 6$ giant impact-type events before it is fully formed. If half of these result in the production of large monolith fragments (as suggested by Stewart \& Leinhardt 2012), then the model-estimated observable transient dust lifetime could be a few times more than the above estimates and hence in reasonably good agreement with the values given at the beginning of this section. If accurate, this suggests that on average there is one terrestrial planet for every star of Solar-mass and above (this does not necessarily mean all stars have terrestrial planets, as some may have multiple and others none). This is in good agreement with the recent determination of the prevalence of rocky bodies orbiting other stars from the Kepler data set (e.g., Burke et al. 2015).

\subsection{Leftover Planetesimals}

Radiometric age-dating of Solar system rocky bodies provides a detailed account of the impact history in the inner parts of our planetary system. Study of impact-reset ${ }^{40} \mathrm{Ar}-{ }^{39} \mathrm{Ar}$ ages in lunar samples and meteorites shows the well-known increase in impactor flux between 3.4 and 4.1 Gyr ago (usually attributed to a Late-Heavy Bombardment phase), but meteorite samples alone also show a spike of reset activity around $\approx 4.5$ Gyr ago (e.g., see Figure 1 of Marchi et al. 2013 and references therein). As discussed in Marchi et al. (2013), these radiometric reset events are often attributed to cooling of the parent body, but substantial evidence suggests that impacts are also important in generating this spike. Impacts in this time frame (ages of 10-100 Myr for the Sun), if indeed sufficiently energetic to reset radiometric geochronometers, likely would have produced significant quantities of dust. Such impacts could in theory be capable of explaining the data presented in Figure 1.

Marchi et al. (2013) postulate that impactors capable of producing the $\approx 4.5 \mathrm{Gyr}$ old ${ }^{40} \mathrm{Ar}-{ }^{39} \mathrm{Ar}$ spike could come from a class of "leftover planetesimals" excited to high orbital eccentricity and inclinations by forming rocky protoplanets (see also Bottke et al. 2007). Bottke et al. (2007) show that a scattered planetesimal population would rapidly deplete, with $\approx 90 \%$ of the sample being removed from the planetary system by the time its host star is $\sim 100 \mathrm{Myr}$ old. The implication is that dust production from this depletion would be dominated by planetesimal-asteroid collisions, although some material should impact the rocky planetary bodies that expelled it. Large rocky planetary bodies are necessary in generating the dust, although they will typically lack an immediate connection to it. As an interesting aside, it is prudent to wonder if such objects could 
also be responsible for late accretion or veneer (e.g., Day et al. 2012; Schlichting et al. 2012).

\subsection{Dynamical Instability}

Solar system formation models (e.g., the Nice model; Gomes et al. 2005; Morbidelli et al. 2005; Tsiganis et al. 2005) predict a phase of outer planetary system reconfiguration wherein the inner planetary system experienced an enhanced influx of planetesimals. This "Late Heavy Bombardment" - which occurred $\approx 600 \mathrm{Myr}$ after the Sun was born - has observational evidence in the cratering record of various inner Solar system rocky objects. It is enticing to consider a similar phase of dynamical instability as capable of driving the exceptionally dusty states of the stars considered in Figure 1 (indeed, Fujiwara et al. 2009, 2012 advance dynamical instabilities to explain the dusty stars they study).

Some studies have shown that it is possible for dynamical instabilities to produce the observed incidence rate of the dustiest main sequence stars (e.g., Bonsor et al. 2013, 2014 and references therein). These simulations also suggest the incidence rate of dusty systems resulting from instabilities and the level of dust being produced by them tend to decrease with increasing stellar age, again in reasonable agreement with the data in Figure 1. But, it is not clear if the level of dust observed can be reproduced as instability models have not yet tracked collisional and dynamical evolution in tandem. New codes should allow coupled simulations and direct tests of these models through predictions for direct observables (e.g., Stark \& Kuchner 2009; Kral et al. 2013).

The implication of this model should it be appropriate is that these systems would host giant planets and planetesimal belts (typically in the outer planetary system) that are experiencing dramatic reconfigurations; rocky Earth-like planets are not immediately necessary.

\section{Conclusion}

There is strong evidence that transient dust production events occur in the inner planetary systems of other stars. Examination of the dustiest main sequence stars as a subset of the debris disk population provides a safe route to selecting those systems where it is clear transient events are occurring and thus to indirectly probing terrestrial planet formation and evolution through the statistics of infrared excess stars. However, more observations, theory development, and simulations are necessary before we can robustly attach these systems to specific phases of planetary system formation or evolution.

\section{References}

Agnor, C. \& Asphaug, E. 2004, ApJL, 613, L157

Agnor, C. B., Canup, R. M., \& Levison, H. F. 1999, Icarus, 142, 219

Asphaug, E., Agnor, C. B., \& Williams, Q. 2006, Nature, 439, 155

Bonsor, A., Raymond, S. N., \& Augereau, J.-C. 2013, MNRAS, 433, 2938

Bonsor, A., Raymond, S. N., Augereau, J.-C., \& Ormel, C. W. 2014, MNRAS, 441, 2380

Bottke, W. F., Levison, H. F., Nesvorný, D., \& Dones, L. 2007, Icarus, 190, 203

Burke, C. J., et al. 2015, ApJ, 809, 8

Day, J. M. D., Walker, R. J., Qin, L., \& Rumble, III, D. 2012, Nature Geoscience, 5, 614

Fujiwara, H., et al. 2009, ApJL, 695, L88

- 2012, ApJL, 749, L29

Genda, H., Kobayashi, H., \& Kokubo, E. 2015, ArXiv e-prints

Gomes, R., Levison, H. F., Tsiganis, K., \& Morbidelli, A. 2005, Nature, 435, 466 
Jackson, A. P. \& Wyatt, M. C. 2012, MNRAS, 425, 657

Kennedy, G. M. \& Wyatt, M. C. 2014, MNRAS, 444, 3164

Kenyon, S. J. \& Bromley, B. C. 2006, AJ, 131, 1837

Kral, Q., Thébault, P., \& Charnoz, S. 2013, A\& A, 558, A121

Marchi, S., et al. 2013, Nature Geoscience, 6, 303

Melis, C., Zuckerman, B., Rhee, J. H., \& Song, I. 2010, ApJL, 717, L57

Melis, C., Zuckerman, B., Rhee, J. H., Song, I., Murphy, S. J., \& Bessell, M. S. 2012, Nature, 487, 74

- 2013, ApJ, 778, 12

— 2014, in IAU Symposium, edited by N. Haghighipour, vol. 293 of IAU Symposium, 273-277

Meng, H. Y. A., et al. 2014, Science, 345, 1032

- 2015, ApJ, 805, 77

Meyer, M. R., et al. 2008, ApJL, 673, L181

Morales, F. Y., et al. 2009, ApJ, 699, 1067

Morbidelli, A., Levison, H. F., Tsiganis, K., \& Gomes, R. 2005, Nature, 435, 462

Olofsson, J., Juhász, A., Henning, T., Mutschke, H., Tamanai, A., Moór, A., \& Ábrahám, P. 2012, A\& $A, \mathbf{5 4 2}, \mathrm{A} 90$

Rhee, J. H., Song, I., \& Zuckerman, B. 2007, ApJ, 671, 616

- 2008, ApJ, 675, 777

Rieke, G. H., et al. 2005, ApJ, 620, 1010

Schlichting, H. E., Warren, P. H., \& Yin, Q.-Z. 2012, ApJ, 752, 8

Schneider, A., Song, I., Melis, C., Zuckerman, B., Bessell, M., Hufford, T., \& Hinkley, S. 2013, ApJ, 777, 78

Song, I., Zuckerman, B., Weinberger, A. J., \& Becklin, E. E. 2005, Nature, 436, 363

Stark, C. C. \& Kuchner, M. J. 2009, ApJ, 707, 543

Stewart, S. T. \& Leinhardt, Z. M. 2012, ApJ, 751, 32

$\mathrm{Su}, \mathrm{K}$. Y. L., et al. 2006, ApJ, 653, 675

Tsiganis, K., Gomes, R., Morbidelli, A., \& Levison, H. F. 2005, Nature, 435, 459

Wyatt, M. C. 2008, ARA $ध A$, 46, 339

Zuckerman, B., Koester, D., Reid, I. N., \& Hünsch, M. 2003, ApJ, 596, 477

Zuckerman, B., Melis, C., Klein, B., Koester, D., \& Jura, M. 2010, ApJ, 722, 725

Zuckerman, B., Melis, C., Rhee, J. H., Schneider, A., \& Song, I. 2012, ApJ, 752, 58 